\title{
PARCS/TRACE COUPLING METHODOLOGY FOR ROD EJECTION ON VVER 1000 REACTOR
}

\author{
MAREK RUŠČÁK ${ }^{a, b, *}$, GUIdO MAZZINI ${ }^{b}$ \\ a Polytechnic University of Catalonia \\ ${ }^{b}$ Research Centre Řež, Hlavni 130, Husinec- ̌̌ě̌, Czech Republic \\ * corresponding author: marek.ruscak@cvrez.cz
}

ABstract. The rod ejection (RE) is a design basis accident in accordance with NUREG-0800 and usually studied using point kinetics. In this paper a methodology and a 3D kinetic model is prepared (PARCS), coupled with a thermal hydraulic system code (TRACE) for simulating this accident scenario for general VVER 1000 technology.

KEYWORDs: PARCS, TRACE, coupling, VVER 1000, safety.

\section{INTRODUCTION}

The technology progress allows the industry to push the accuracy of calculations to the new level. Alongside with the progress, there is an approach to simplicity. The current trend is to couple already existing codes to enable simultaneous complex scenarios and sub-sequential analysis. The thermo-hydraulic system code TRACE is the next generation code being a successor to RELAP, both of which however have the capability of coupling with the neutronics code PARCS.

The PARCS/TRACE coupling for full VVER 1000 reactor has never been done before in the Czech Republic with the cross sections generated by SCALE/TRITON. Therefore a methodology had to be developed, which proved to be a challenge on its own regarding the lack of documentation for coupling models with hexagonal geometries. Most of the geometrical, material and structural data are taken from a reference VVER 1000 [1].

It is important to note, that results in this paper are not final and much longer process of model development will be required in order to submit the coupled model for validation. Main objective of this paper is to demonstrate in a practical example of threedimensional accident scenario of VVER 1000 reactor an acquisition of PARCS code for 3D reactor kinetics and its coupling with system thermo-hydraulic code TRACE.

\section{PARCS CODE DESCRIPTION}

PARCS (Purdue Reactor Core Simulator) is a threedimensional (3D) reactor core simulator which solves the steady-state and time-dependent, multi-group neutron diffusion and low order transport equations in orthogonal and non-orthogonal geometries. PARCS can be coupled directly to the thermal-hydraulics system code TRACE which provides the temperature and flow field information to PARCS during the transient calculations via the few group cross sections. PARCS is available as a standalone code for performing calculations which do not require coupling to TRACE or RELAP5. PARCS can also be coupled to the RELAP5 systems code. A separate code module, GENPMAXS, is used to process the cross sections generated by lattice physics codes such as TRITON (SCALE 6.1.2), HELIOS, or SERPENT into the PMAXS format that can be read by PARCS.

PARCS is composed of several cards such as CNTL (control), XSEC (cross-sections) and so on. PARCS code is only concerned about neutronic definitions, most importantly the macroscopic cross sections, radial and axial fuel assembly maps, mashing and other parameters [2].

The most recent version of the PARCS code is 3.2 (November 2015). Although PARCS is a very good tool for square geometry core lattice, there are still limitations concerning hexagonal geometry. By the fall the Code Application Maintenance Program (CAMP) of 2015 it is not possible to calculate pin power of hexagonal assemblies. Also presented at the CAMP by Dr. Guido Mazzini and by the author there is a limitation using gadolinium as a burnable absorber in the core in TRACE. There several additional issues concerning the coupling procedure, mainly due to a lack of proper guiding material provided by the code authors. In numerous cases impact and importance of several parameters can only be found by performing a sensitivity analysis on them, which requires significant amounts of time and therefore it is difficult to build a near-perfect model 3 .

\section{TRACE CODE DESCRIPTION}

The TRAC/RELAP Advanced Computational Engine (TRACE - formerly called TRAC-M) is the latest in a series of advanced, best-estimate reactor systems codes developed under U.S. Nuclear Regulatory Commission for analysing transient and steady-state neutronic-thermal-hydraulic behaviour in light water reactors. It is the product of a long term effort to com- 
bine the capabilities of the NRC's four main systems codes (TRAC-P, TRAC-B, RELAP5 and RAMONA) into one modernized computational tool. It is able to analyse large/small break Loss of Coolant Accidents (LOCAs) and other system transients in both pressurized water reactors (PWRs) and boiling water reactors (BWRs). The version used in this analyses is TRACE v5 patch 4 released in 2014 .

For the user point of view, there are three major steps in performing a calculation in TRACE and those are: input processing, initialization and results. During the input processing, TRACE checks the user model and checks for errors. If the model passes the processing, it is initialized and again checks for possible boundary error as well as the model will be prepared for calculation. Once the calculation is finished, there are several output files, among which there is the actual output, a restart file and a binary file containing all data to be read by apt plot. This process is shown in the Figure 1 [4].

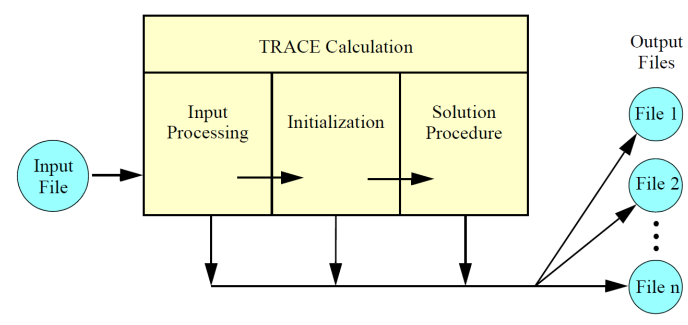

FIgURE 1. Phases of a TRACE calculation.

\section{SCALE CODE DESCRIPTION}

The SCALE code package is a comprehensive modelling and simulation suite for nuclear safety analysis and design. SCALE provides a "plug-and-play" framework with several computational modules including three deterministic and three Monte Carlo radiation transport solvers that are selected based on the desired solution strategy. SCALE includes current nuclear data libraries and problem-dependent processing tools for continuous-energy and multigroup neutronics calculations, multigroup coupled neutron-gamma calculations, as well as activation and decay calculations. In particular the TRITON Module is used for the generation of cross-sections useful for PARCS code. It is a control module in computation code SCALE 6.1.2. It uses several computational sequences like CENTRM, NEWT, BONAMI, etc., that are depending on user's requirements. TRITON performs 2-D calculations for square and hexagonal geometry of the fuel assembly. Its main computational features are cross-section calculation and isotopic compositions of the fuel assembly. In the CENTRM-based discrete ordinates ( $\mathrm{SN}$ ) option, the Bondarenko self-shielding method is used to determine the problem-dependent multigroup cross-sections in the unresolved resonance energy range. The unresolved resonance calculation is performed by the BONAMI functional module. For the resolved resonance energy range, the CENTRM functional module is used to determine the $1 \mathrm{D}$ pointwise (105 energy groups) flux solution using the SN method [5].

\section{Coupling procedure}

Coupling procedure differs greatly with the lattice of the reactor. For square pitch all the user needs are PARCS and TRACE inputs and graphical interface called SNAP in order to use the auto mapping feature. This auto mapping procedure generates a "map" file to be run with the coupled calculation. This automatic process is not however available for this case. Therefore in case of hexagonal pitch this procedure has to be done manually. This means manually assigning each neutronic control volume (mesh) to the one of TRACE's hydraulic and heat structure meshes. To do this by hand would require a substantial amount of time and this is why to achieve mapping a secondary script done in MATLAB is used.

The general structure of the script has four main sections. The general constants such as number of radial rows or axial planes, number of assemblies, number of planes and the vessel ID defined in the TRACE model. In the second section there are the radial definitions of both PARCS and TRACE. First there is PARCS radial numbering (given by stand alone PARCS output) and then the PARCS radial weight map (0.0-1.0) where on azimuthal boundaries the assembly weight is 0.5 and doubled (two assemblies by 0.5 instead of one by 1.0) and the central node is divided into 6 by $1 / 6$. The TRACE radial TH map and a Heat Structure (HS) map are defined according to a logic set in TRACE.

The third section of the mapping script concerns the axial meshing and mapping. It is important to note, that the number of axial levels has to be same as in the geometry definition in both PARCS and TRACE as well as the node numbering. Usually the vessel component in TRACE has 1 to $n$ nodes where the core $(k)$ itself occupies only $n-k$ nodes and the numbering has to respect the numbering in TRACE for the core. The mapping file includes a table in the following columns; vessel ID, TRACE radial ID, TRACE axial ID, PARCS radial and multiplication of radial and axial weights.

After the mapping file is generated it is included with the PARCS and TRACE inputs in order for the codes to recognize the geometry. In Figure 2 there is a visualization of the mapping process, where colours correspond to individual sectors. Strictly speaking the nodal definitions in both PARC (core) and TRACE (TH and HS) are put together.

The sequence of calculation is shown in Figure 3 on page 65. In this work there are two sets of cross sections generated by the SCALE and SERPENT 2 codes. Later on the results will show the differences between the two and possible ways of improvement. Once the cross sections are implemented with the PARCS code, 


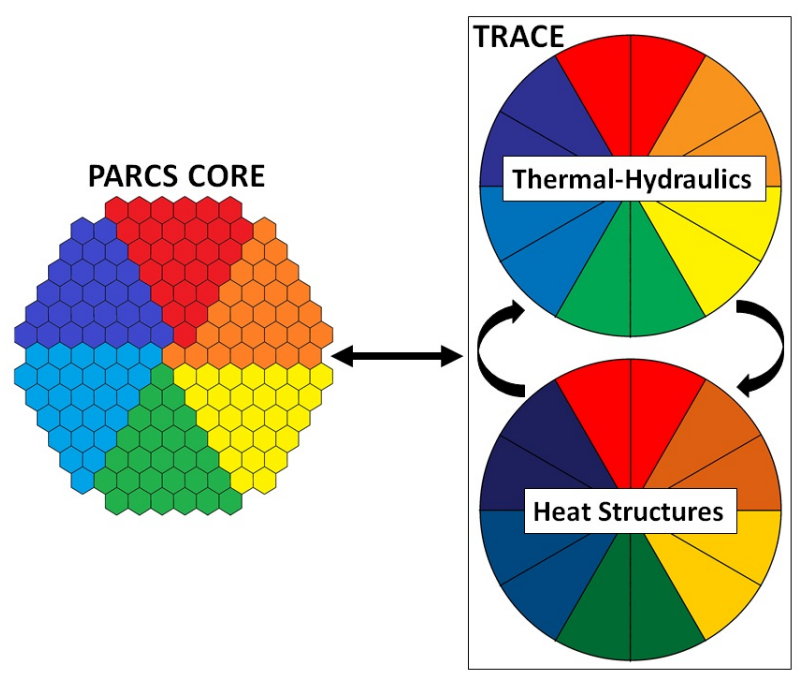

FiguRE 2. Mapping procedure.

geometry definitions of PARCS and TRACE codes have to be the same. After ensuring that the geometry is same in either code, it is possible to begin the coupling process, which begins with mapping of the geometry definitions. In order to couple PARCS and TRACE there are four files needed. The PARCS and TRACE inputs (in the diagram they are shown as NAME.inp) and TRACE Stand Alone (SA) restart file are generated by the Stand Alone (SA) TRACE and of course the mapping file. With these files it is possible to run Steady State (SS) Coupled (C) calculation. This calculation outputs several files including the restart files of both PARCS and TRACE, which are necessary for transient ( $\mathrm{Tr}$ ) calculation. The SS, $\mathrm{C}$ restart files are then used for the Tr, C calculation along with the transient inputs of PARCS and TRACE.

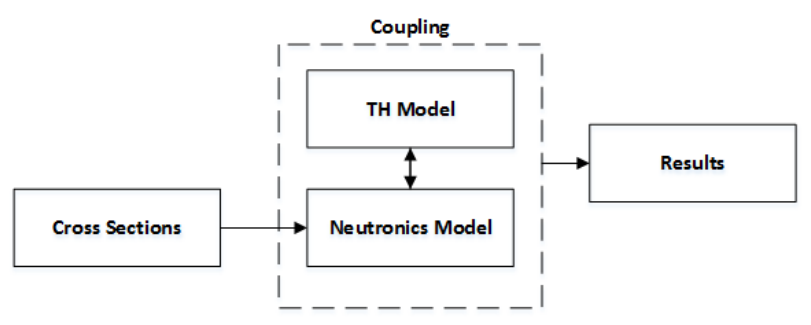

Figure 3. Calculation Process.

\section{Model Description}

\subsection{SCALE}

The model of fuel assembly is created by TRITON in SCALE 6.1.2 and the geometrical and material parameters from a VVER 1000 type NPP. There are six types of fuel assemblies with different enrichment as shown in the Table 1 (page 66). In the first step it is necessary to define several parameters of the fuel, such as density, temperature and enrichment as well as cladding, which consist of $99 \% \mathrm{Zr}$ and $1 \% \mathrm{Nb}$.
Moderator is comprised from main an additional materials. The main material is water $\left(\mathrm{H}_{2} \mathrm{O}\right)$ with density of $0.73 \mathrm{~g} / \mathrm{cm}^{3}$, temperature $575 \mathrm{~K}$ and the additional material is boron with $1065 \mathrm{ppm}$. Inside the fuel pellets there are the central holes filled with helium. The geometrical definition and parameters of the fuel assemblies and fuel pellets are defined as following. Simulation of active length of fuel assembly as infinite because calculation code TRITON computes only 2D (radial) geometry. The inputs are shown in Figure 4 and Figure 5 on page 65. Fuel assembly consists of nine nodes and each node has power level that depends on the active length power distribution. The analyses in SCALE are performed with average power and temperature distribution, also the calculation are done at time 0 , practically after refuelling without the effect of xenon and samarium. The calculations are based on ENDF/B-VII.0 in 238 groups. The plots below are a visualization of defined geometry, where different colours represent different materials.

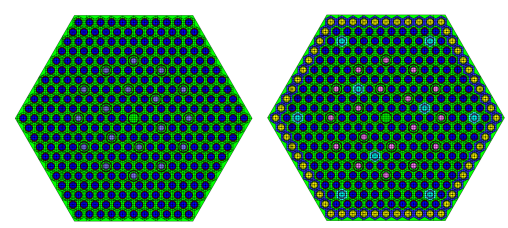

Figure 4. Inputs of F130/F200 (left) and inputs of FF36G9/FF40G9 (right).

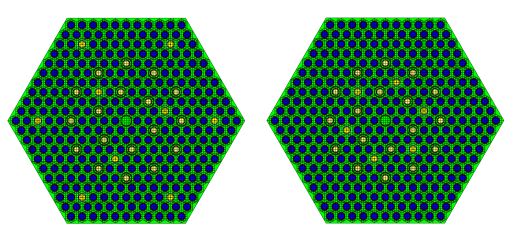

Figure 5. Input of F30G9 (left) and input of F40G6 (right).

There are five branches considered plus a reference one. The branch information is in the Table 2 on page 66. These branches are used for calculation of the differential cross sections. The branches are fuel temperature (Tf), moderator temperature (Tm), moderator density (Dm), control rod position (CR) and boron concentration (Sb).

\subsection{PARCS}

The neutronic model of VVER 1000 in the PARCS code is fairly simple and short, compared to system codes or MC codes, such as MCNP.

Most of the parameters in the model are kept default. The only important parameter specified is the nodal kernel used, in this case the triangular polynomial expansion nodal (TPEN) method. This method first decouples the 3-D defined geometry into 2-D axial and radial that are coupled through transverseleakages. The radial and axial configuration as well as 


\begin{tabular}{cccccc}
\hline & Fuel 1 [\%] & Fuel 2 [\%] & Fuel Gd [\%] & Gd [\%] & Gd pins [-] \\
\hline F200 & 2.0 & - & - & - & - \\
F130 & 1.3 & - & - & - & - \\
F40G6 & 4.0 & - & 3.3 & 5 & 6 \\
F30G9 & 3.0 & - & 2.4 & 5 & 9 \\
FF36G9 & 3.6 & 3.3 & 3.3 & 5 & 9 \\
FF40G9 & 4.0 & 3.6 & 3.3 & 5 & 9 \\
\hline
\end{tabular}

TABLE 1. Fuel enrichment description.

\begin{tabular}{cccccc}
\hline & Tf $[\mathbf{K}]$ & Tm [K] & Dm [g/cm3] & CR & Sb [ppm] \\
\hline Reference Branch & 980 & 575 & 0.73 & Out & 1065 \\
Branch 1 & 980 & 575 & 0.73 & In & 1065 \\
Branch 2 & 980 & 575 & 0.73 & Out & 565 \\
Branch 3 & 980 & 575 & 0.83 & Out & 1065 \\
Branch 4 & 980 & 675 & 0.73 & Out & 1065 \\
Branch 5 & 1080 & 575 & 0.73 & Out & 1065 \\
\hline
\end{tabular}

TABLE 2. SCALE Branch structure.

control rod banks positions are also defined. The control rod configuration is set so the shut down clusters are fully withdrawn from the core, and the control clusters are fully inserted. Core has to have same dimensions and meshing as in the TRACE model, in order to couple. Therefore there are 14 axial nodes and 8 radial rings as shown in Figure 6 on page 66 and Table 4 on page 67. The description of Figure 6 is shown in Table 3 on page 66 [1, 6].

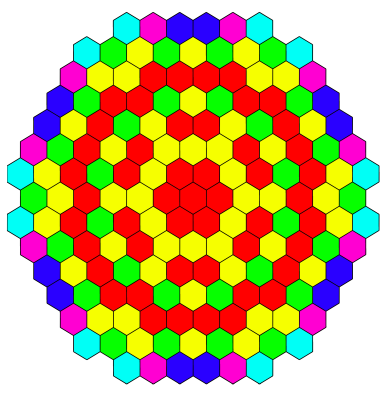

Figure 6. Core layout.

\begin{tabular}{cc}
\hline Colour & FA type \\
\hline Red & F130 \\
Yellow & F200 \\
Green & F30G9 \\
Azur & F40G6 \\
Blue & FF36G9 \\
Purple & FF40G9 \\
\hline
\end{tabular}

TABLE 3. Colour description for core layout.

The general configuration of control and SCRAM banks is shown in Figure 7 on page 66 where different colours indicate different groups.

This model however does not include reflector, due to the complexity of implementation. Whole TRACE

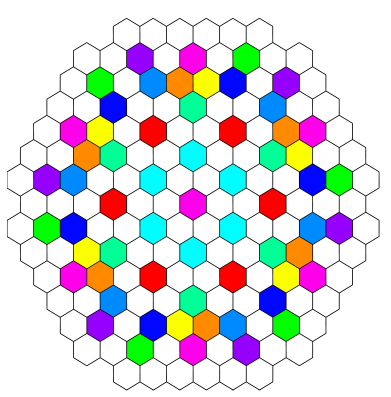

Figure 7. Bank layout.

model would have to be changed in order to include one additional ring containing the reflector.

\subsection{TRACE}

The VVER 1000 model developed by CVR was originally prepared for Relap5Mod3.3 code and subsequently converted for TRACE V5.0 code. It represents the primary and secondary systems, as well as the safety components of the plant. The model of the primary circuit consists first of the vessel component representing the reactor pressure vessel (RPV) as illustrated in Figure 8 on page 67 Radially, three zones nodalization scheme is adopted with two inner zones representing the lower plenum, the core, and the upper plenum. Third (outer) zone models the downcomer. Axially, 37 zones are modelled with 14 of them representing the active core. Further, the model of the primary circuit consists of models of each individual primary system loop subdivided into hot leg pipes, horizontal steam generators hot and cold collectors and heat exchange tube bundles, loop seal pipes, reactor coolant pumps, and cold legs. To complete the primary circuit model the pressurizer, pressurizer surge and spray lines are modelled. The pressurizer power operated relive valve and safety valves are sim- 
ulated. TRACE V5.0 pipe and valve components are used to model the secondary system steam lines up to the turbine. Steam dump valves and steam generator safety valves are simulated individually. Feedwater system model consists of the pipes and valves located in the containment. The plant model further contains the control components and trips to simulate actions of safety grade portions of I\&C.

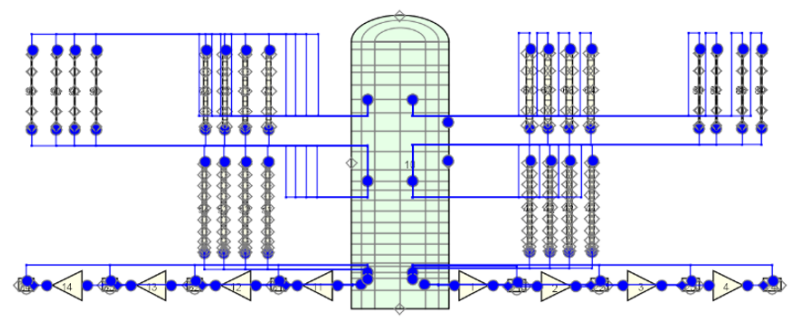

FIgURE 8. TRACE RPV model nodalization.

The basic idea of this model development was to develop a tool for the licensing audit calculations of DBA events included in Chapter 15 of the SAR [7. As such, the model was systematically validated against the number of plant start up tests including the loss of flow (RCP trips), loss of feedwater, etc. With this, and considering that Best-estimate codes are more accurate than severe accident codes in phases before core degradation started, the TRACE V5.0 model is a reference model for initial phases of the transients simulated [8].

The transient model is a reduced version of the steady state input, in order to reduce the computational costs. Also it includes the Small Break Loss Of Coolant Accident (SBLOCA), (trip number 1005) which occurs at the point of ejection when the upper part of the control mechanism breaks and is ejected from the pressure vessel. This trip is set manually and the setpoint is time.

The axial core control volume distribution is shown in the Table 4 on page 67. This table does not include all RPV nodes, but only the active core, since the entire nodalization is not essential for the purposes of this work.

The time steps are crucial part of the model, especially when coupling. The most important parameter is that the time step in TRACE should be about three orders greater then the timestep set in PARCS, but small enough to allow the calculation to perform [4]. From experience however, TRACE terminates due to high time step, yet after rigorous sensitivity analysis the problem is not solved simply by reducing the time step even further. This particular problem will be one of a subjects of further study after this work.

\section{RESUlTs}

The results suffer from several code-based issues. Probably the biggest impact has so called null factor, which acts as a balancing reactivity factor. In the case during the transient calculation, if the k-effective differs

\begin{tabular}{cc}
\hline Axial level & Mesh size \\
\hline Axial level 14 & 46.3 \\
Axial level 13 & 25.5 \\
Axial level 12 & 25.5 \\
Axial level 11 & 25.5 \\
Axial level 10 & 25.5 \\
Axial level 9 & 25.5 \\
Axial level 8 & 25.5 \\
Axial level 7 & 25.5 \\
Axial level 6 & 25.5 \\
Axial level 5 & 25.5 \\
Axial level 4 & 25.5 \\
Axial level 3 & 37.73 \\
Axial level 2 & 13.27 \\
Axial level 1 & 29.55 \\
\hline
\end{tabular}

TABLE 4. TRACE axial distribution.

from 1 , this null factor acts to balance reactivities to achieve $\mathrm{k}$-eff $=1$. The bigger the difference between $\mathrm{k}$-effective actual and 1, the bigger the null factor. Another possible reason for these oscillations are the cross sections themselves and more tuning may be needed. This major reason is the lack of boron reactivity feedback, which still remains to be a problem. This is suspected to be the reason why the reactivity stabilizes at $0.2 \$$ (see Figure 17). Lastly the minor source of these oscillations is the instability transition of TRACE from steady state to transient calculation. Because the access to the source code of either PARCS or TRACE is limited, the only way of addressing these uncertainties is through benchmarking with existing results.

When the control bank ejects, there is about $1 \$$ of positive reactivity inserted to the system, resulting higher neutron flux, this resulting in higher fuel temperature (Figure 19) and also causing a rise of the Doppler temperature. Therefore the Doppler effect introduces negative reactivity to the system, decreasing power. This heat is being transferred to the coolant, resulting in increase of coolant temperature (Figure 18). When the temperature of the coolant (in this case light water) increases, the density decreases (Figure 21) resulting in lower moderation hence decrease in flux, reactivity and power. This is the general behaviour, that occurs in all the peaks. The first peak, (Figure 12) is so small, because the localised rise in coolant temperature is stopped when it is cooled down by rest of the coolant in the core. However quickly after the power start rising again and the whole process described above occurs again, however much more slowly. The final oscillations show simple balancing between moderator density and moderator temperature. By comparing the results with Chapter 15 of Final Safety Analysis Report of an existing VVER 1000, which unfortunately cannot be shown, the results are fairly comparable also with the results of 9 .

By examining the flux behaviour we can see, that 


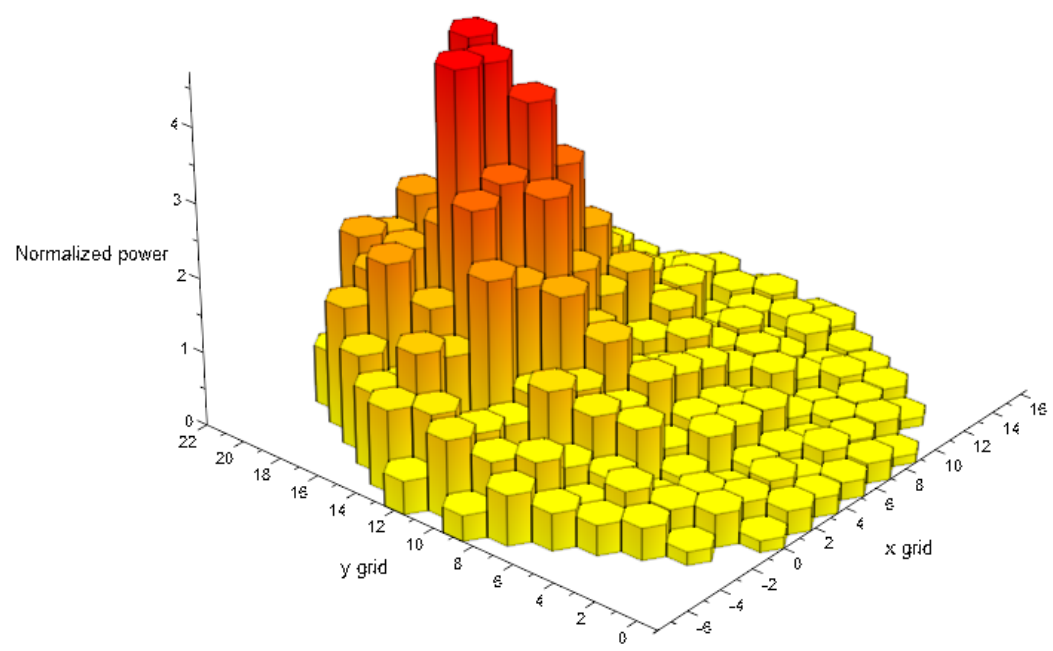

Figure 9. Averaged radial normalized power profile.

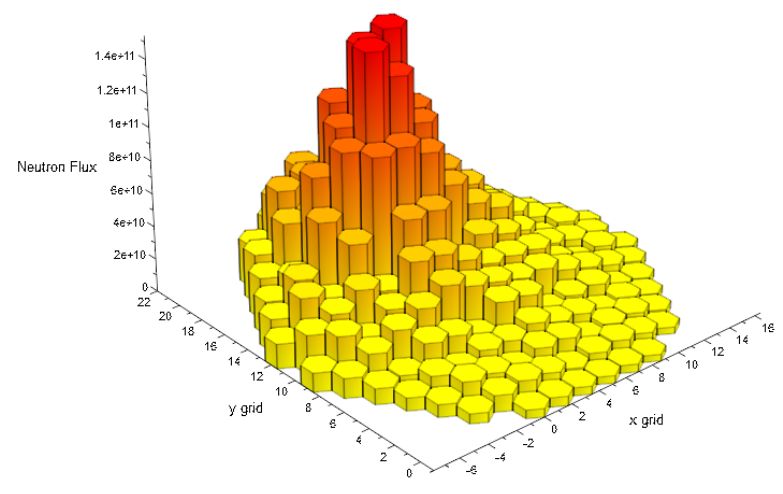

Figure 10. Averaged thermal flux.

in 100 seconds the flux increases during the transient. This can be seen in Figure 10 on page 68 . The flux shape is actually one of the major reasons why to use 3D calculations. By examining the flux plots below, most of the core has fairly low flux, in comparison to the area around the ejection point. By using the standard approach of point kinetics, the results would be averaged over the whole core, therefore lower then the actual highest peak.

In the meantime, when the control bank ejects, the driving mechanism breaks and is ejected from the pressure vessel, resulting in a small break LOCA of a $50 \mathrm{~mm}$ diameter. The loss of coolant is represented by the mass flow in the Figure 22, however for the duration of the transient, SB LOCA has very little effect as seen from the system pressure in Figure 23. There are pressure oscillations close after ejection, however it stabilizes at the end of the transient.

In order to calculate the main parameter, which is amount of energy per gram (cal/g), it is necessary to integrate over one second the relative power in the

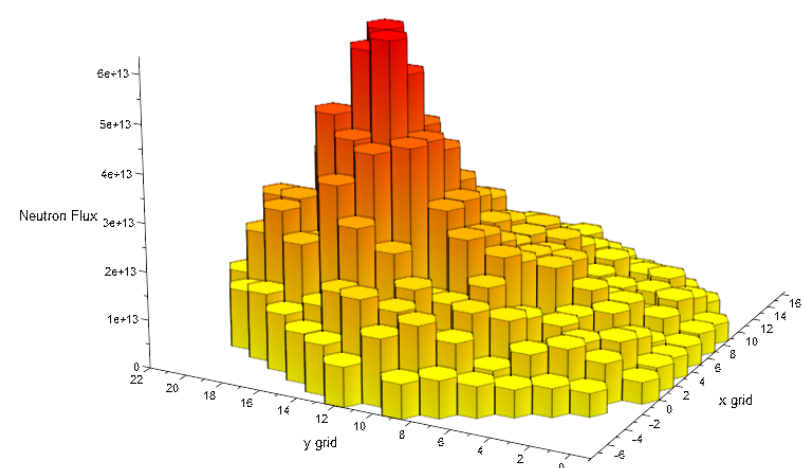

Figure 11. Averaged thermal flux after $100 \mathrm{~s}$.

ejection point and then multiply by the total power at peak [W] over number of assemblies $(\mathrm{Na}, 163)$. Using the hot channel radial peak factor $(P F) 1.29$ (given through internal communication with VVER $1000 \mathrm{FA}$ specialists) and dividing the total weight of fuel (approximately $500 \mathrm{~kg}$ ) in one assembly by the number of pins (311). The general calculation is following, having time step $(t)[\mathrm{s}]$, average power $(P)$ $[\mathrm{W}]$ and normalized power at ejection point $(R p)[-]$. First it is important to calculate power in Watts $(W r)$ for the ejection point which is $W r=P \cdot R p \cdot \frac{P F}{N a}$.

After that by integrating the $W r$ curve per time and so getting the energy per time $[\mathrm{J}]$, it is necessary to convert to calories $(1 \mathrm{~J}=0.239 \mathrm{cal})$ and divide by weight of fuel in one pin in grams. The expected results should be similar to 9 ] which is $70.5 \mathrm{cal} / \mathrm{g}$. The results are shown in Figure 13 and it is possible to see, that the maximum value is $75 \mathrm{cal} / \mathrm{g}$ and therefore below the limit of $200 \mathrm{cal} / \mathrm{g}$ and also that it is reasonably similar to the results of [9], considering the differences in tested reactors. 
Reactor Power

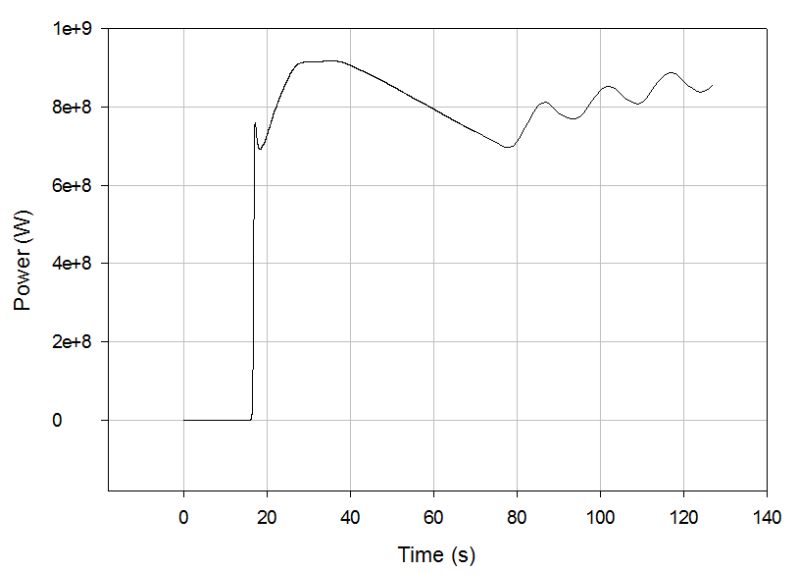

Figure 12. Core power.

Maximum Energy Deposited in Fuel Pin

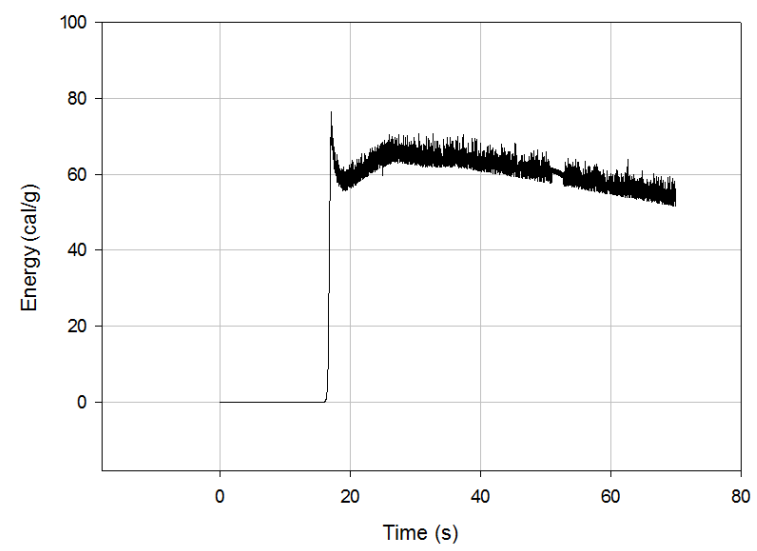

Figure 13. Maximum energy deposited in fuel pin.

Flux Cross Section at Ejection Point

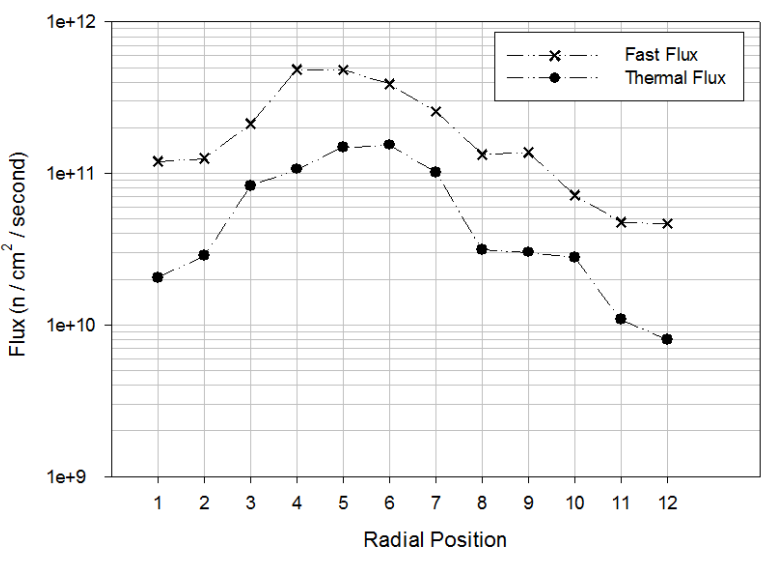

FigURE 14. Flux profile cross section at ejection point.
Flux Cross Section at Ejection Point

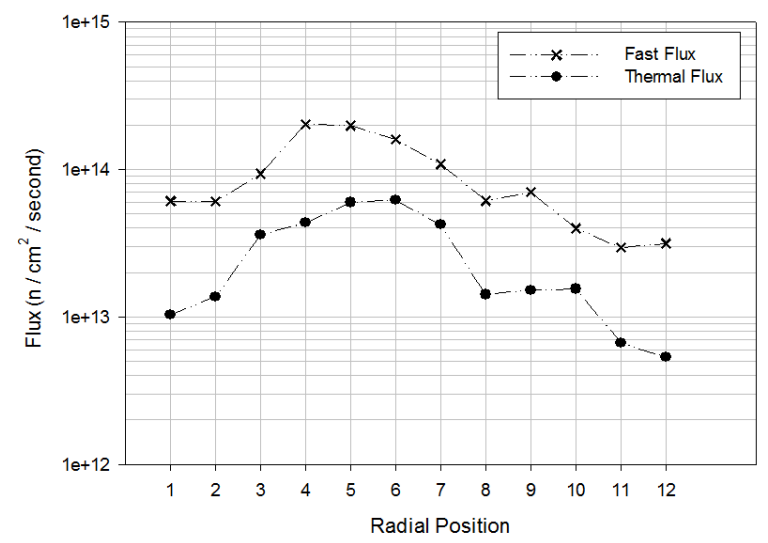

FIGURE 15. Flux profile cross section at ejection point after $100 \mathrm{~s}$.

Axial Power Distribution

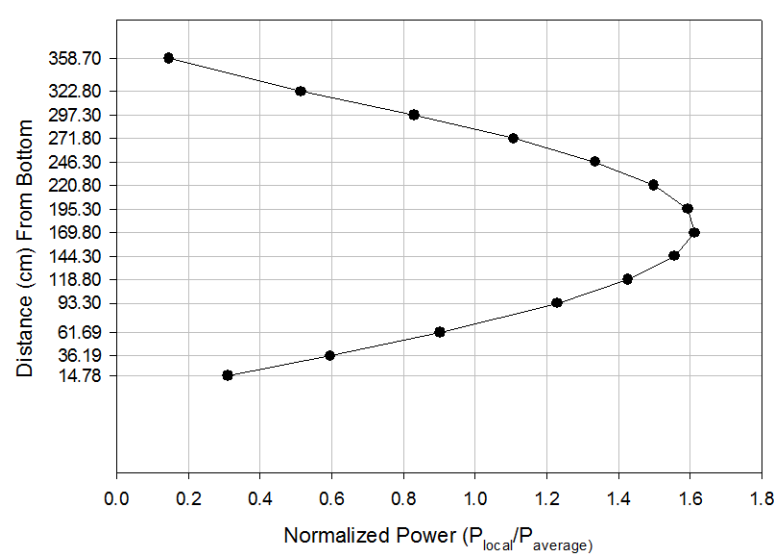

FiguRE 16. Axial normalized power.

Reactivity

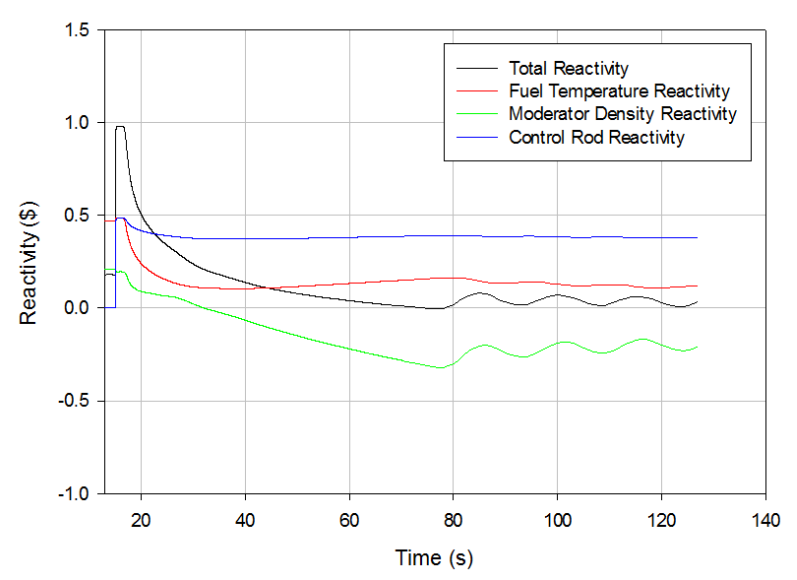

Figure 17. Reactivity. 
Coolant Temperature at Ejection Point

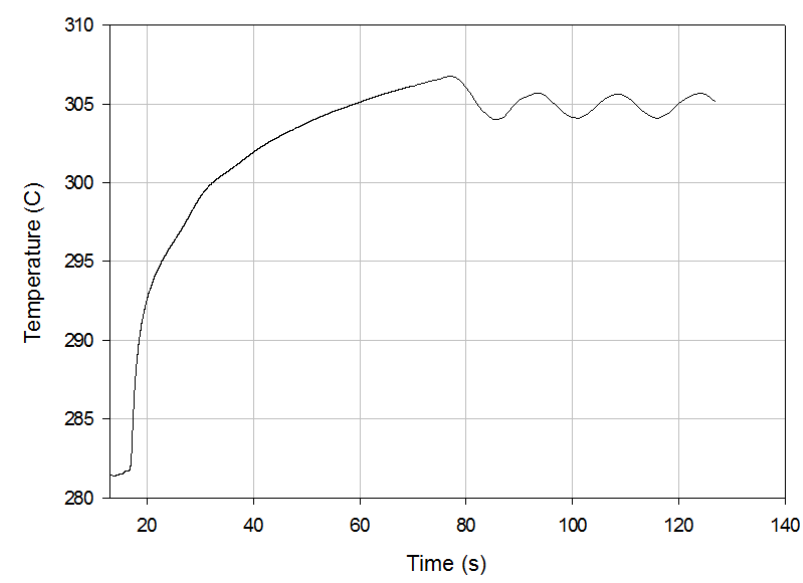

Figure 18. Coolant temperature at ejection point.

Fuel Temperature at Ejection Point

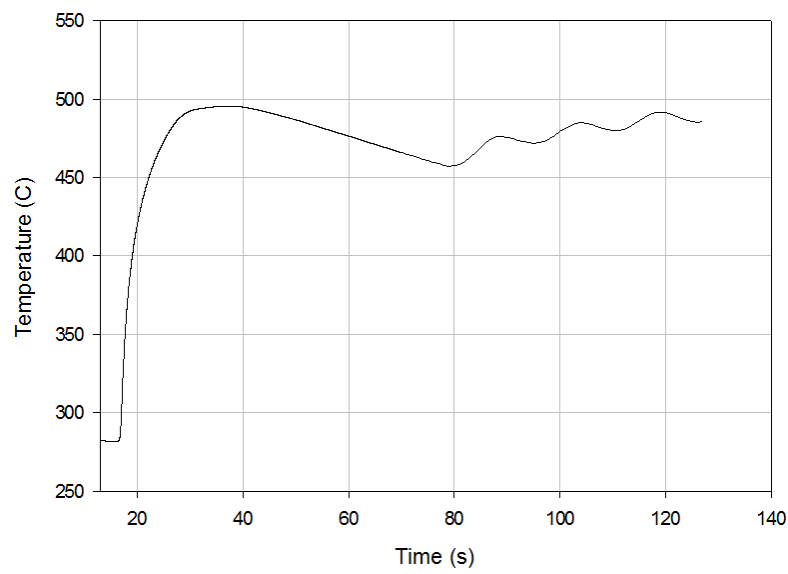

Figure 19. Fuel temperature at ejection point.

Maximum Hot Rod Temperature

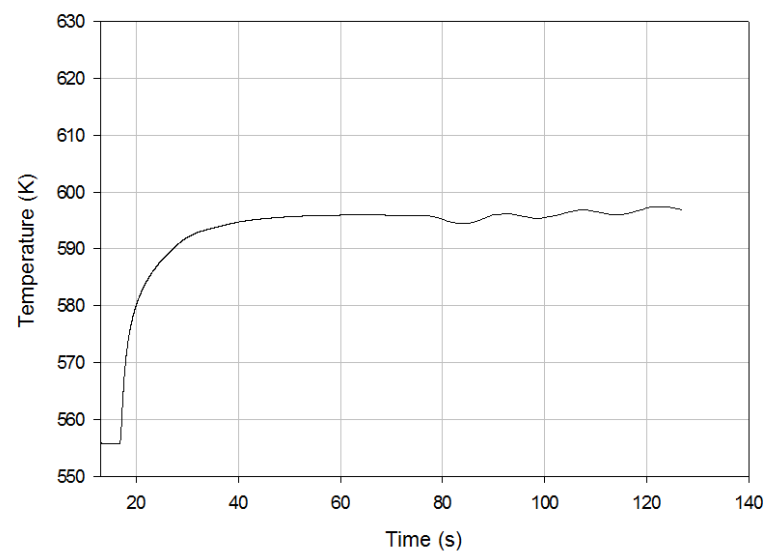

Figure 20. Maximal hot rod temperature.
Coolant Density at Ejection Point

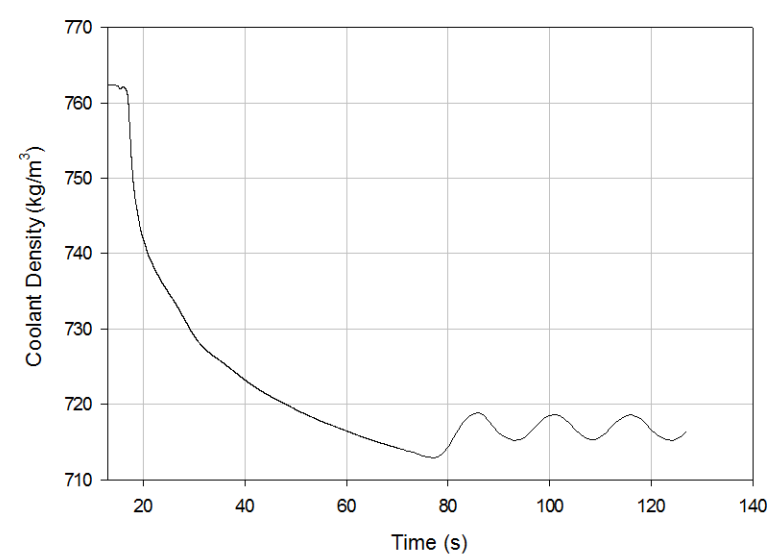

Figure 21. Coolant density at ejection point.

Liquid Mass Flow Through Break

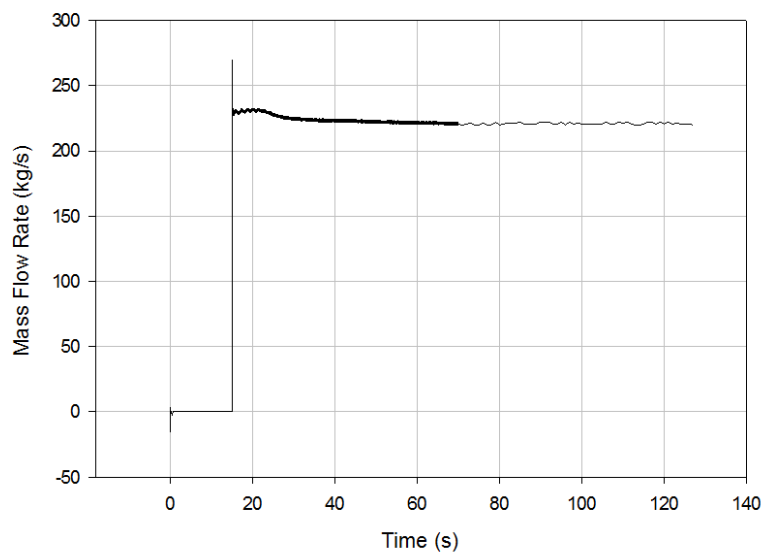

Figure 22. Mass flow through the break.

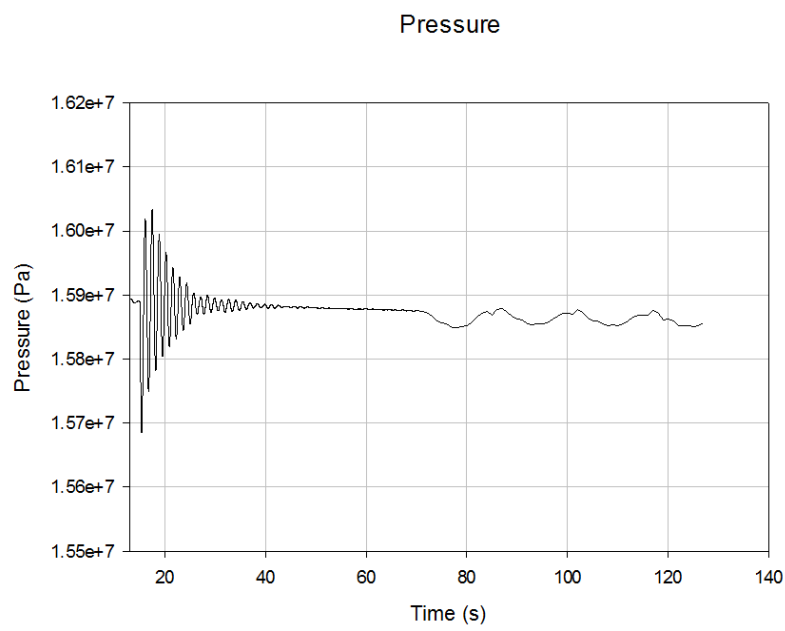

Figure 23. System Pressure. 


\section{Conclusion}

The rod ejection accident scenario is a Reactivity Initiated Accident and is also considered a Design Basis Accident (DBA). The general approach is to improve the existing models and methodologies in order to increase the precision of results. This goal is being approached from two sides, first to couple different codes (neutronics with thermal-hydraulics and also with material codes) together and second to transition from $1 \mathrm{D}$ calculations to $3 \mathrm{D}$. This is increasingly possible with the increase in computer power, which allows to run much more complex calculations.

There were two main goals to this paper. First, to establish a methodology for coupling PARCS with TRACE for reactor with hexagonal lattice and second to run a rod ejection scenario on this coupled model.The general process was to create homogenized macroscopic cross sections for six different fuel assembly types, specifications of which were provided by the Research Centre Rez and are considered confidential. The homogenized cross sections were calculated using a SCALE/TRITON code. After the cross sections were implemented into the PARCS models, the PARCS geometry definition had to be mapped with the one of TRACE. After that, a reference steady state coupled calculation was performed for each scenario separately. When the individual steady state calculations were done, the resulting restart data were inserted into the transient models.

There were several challenges when coupling these codes for hexagonal lattice. The major one, which costed the most time is a lack of a proper guide on how to perform coupling without using graphical interface SNAP, which does not support hexagonal lattice. This issue is currently being solved with the authors of the code and hopefully a better user guide will be available in next years. Second difficulty was the absence of auto-mapping function for hexagonal lattice, which had to be substituted by a MATLAB script. This can also be a source of uncertainty, because it is difficult to be sure, that the mapping was done correctly. Lastly the code itself has a significant setback for the rod ejection calculation, which is a lack of pin power calculation for hexagonal lattice. Because of this, the power is averaged over the whole assembly and therefore is lower, than the actual value. This can be however corrected using the peak factor as done in this paper. Currently there are alternative neutronic codes, that can be coupled with TRACE, for example DYN3D, which can be coupled not only to TRACE, but also with RELAP and ATHLET codes.

In the end, the future work to be done is firstly to improve the user guide for coupling PARCS with TRACE for hexagonal lattice. The work done in this paper will undergo further tuning and a number of sensitivity analyses will be performed to achieve better results. Firstly a database of cross sections will be created for multiple steps in the burnup cycle and secondly the TRACE model will have to be further modified for coupling.

\section{ACKNOWLEDGEMENTS}

I wish to thank Ing. Miroslav Hrehor for allowing me to perform my internship at the Research Centre Rez and do my master thesis. Also I wish to thank Ing. Milos Kyncl for providing and assisting with the TRACE model and to Ing. Bruno Miglierini and Ing. Vit Kopecek for technical help.

I would like to also thank the U.S. NRC for helping me with several technical issues with the coupling procedure.

\section{REFERENCES}

[1] M. Ruscak. Simulation of reactor kinetics in nuclear power plant using simulator PARCS. Bachelor's thesis, University of Hradec Kralové, 2014.

[2] B. Miglierini, M. Ruscak, G. Mazzini. Practical acquiring of the PARCS code for $3 \mathrm{D}$ analysis of neutronic behavior of VVER1000/V320. VVER 2013 Conference, 2013.

[3] T. Downar, Y. Xu, V. Seker. PARCS v3.2, U.S. NRC Core Neutronics Simulator. U.S. Nuclear Regulatory Commision, 2014. UM-NERS-09-0001.

[4] U.S. Nuclear Regulatory Commision. TRACE V5.840 Manual. Division of Safety Analysis, Office of Nuclear Regulatory Research, U.S. Nuclear Regulatory Commission, Washington, DC.

[5] Radiation Safety Information Computational Center at Oak Ridge National Laboratory. A Comprehensive Modeling and Simulation Suite for Nuclear Safety Analysis and Design. Oak Ridge National Laboratory, 2011. ORNL/TM-2005/39.

[6] B. Miglierini, G. Mazzini, M. Ruscak. 3d neutronic analysis of VVER1000/V-320 using PARCS code. In Proccedings of the 2014 15th International Scientific Conference on Electric Power Engineering (EPE). 2014. DOI:10.1109/EPE.2014.6839473.

[7] U.S. Nuclear Regulatory Commision. NUREG-0800, Standard Review Plan for the Review of Safety Analysis Reports for Nuclear Power Plants, LWR Edition. U.S. Nuclear Regulatory Commision, 2007.

[8] M. R. Guido Mazzini, Mylos Kyncl. Analyses of feed water trip with SBO sequence of VVER1000 reactor. Journal of Nuclear Engineering and Radiation Science 2016. DOI:10.1115/1.4034144.

[9] J. V. Javier Riverola, Tomas Nunez. Realistic and conservative rod ejection simulation in a PWR core at HZP, EOC with coupled PARCS and RELAP. In Proceedings of the 2004 International Meeting on LWR Fuel Performance, Orlando, Florida. 2004. 